\title{
Efficacy-Driven Quality Control Platform for Chinese Herbal Medicine
}

\author{
Linda LD Zhong ${ }^{1}$, Dong-Dong Hu${ }^{2}$, Hong-Xi Xu ${ }^{2}$, Quan-Bin Han ${ }^{1}$ and Zhao-Xiang Bian ${ }^{1 *}$
}

${ }^{1}$ School of Chinese Medicine, Hong Kong Baptist University, Hong Kong SAR

${ }^{2}$ School of Pharmacy, Shanghai University of Traditional Chinese Medicine, PR China

\section{Introduction}

Chinese herbal medicine (CHM) has been used in China and Eastern Asia more than two thousand years, and is becoming more and more popular in recent years. Currently, CHM is rapidly gaining attention in the West as sources of new drugs, dietary supplements and functional foods. However, the lack of consistent manufacturing processes, quality standards, scientific, and the validation of efficacy and safety impede worldwide acceptance of CHM [1]. Particular attention has been focused on efficacy and safety of CHM and quality control is a key part for the drug discovery, especially for the proprietary Chinese medicine [2-4].

\section{Current Quality Control System of CHM and Weakness}

The quality control of CHM is always a key issue for the clinical usage, and also is a secure for high quality of clinical trials, especially in the consistency and controllability [5,6]. Great progress has been made in the quality control of CHM due to modern separation and characterization techniques. Briefly, it can be divided into three stages. The first stage was the usage of identification of CHM based on material medical's morphology, one or two markers' thin-layer chromatography (TLC) [7]. The morphological identification was described in the 1953 and 1963 editions of the Chinese Pharmacopeia. Since 1985, TLC has been employed for the qualitative evaluation of CHM [8]. Morphological identification can give a quick identification of $\mathrm{CHM}$; and TLC method has the advantages of operating conveniently, quick identification and preliminary quantification of marker compounds. However, morphological identification cannot explore the substantial basis of CHM; while TLC also has the weaknesses of limitation in multiple compounds detection, low selectivity in quantification. CHM is a multicomponent combination, so just use TLC method for several marker compounds' quality control cannot reflect the real quality of CHM. Nevertheless, the chosen of marker compounds are mostly based on main compounds in CHM, which may have no relevance with pharmacological activity.

Based on these weaknesses, Researchers started to focus on multiple active compounds analysis method. So the second stage was the application of comprehensive methods which reflect the characteristics of multiple targets and synergistic action of $\mathrm{CHM}$ using fingerprint (including chromatographic and spectroscopic ones, contain TLC, HPLC, GC, CE, IR, NIR, NMR, DNA, GC-MS, LC-MS, LC-DADMSn, and LC-DAD-NMR) $[9,10]$. It presents the entire composition information and fulfills the TCM characteristics. Therefore, fingerprint analysis has been internationally accepted for the quality control of herbal medicines [8]. To combine chemical character with bioactivity, bio-fingerprint and metabolic fingerprint have also been used for directly selecting the marker constituents for quality control [11]. However, these marker components were selected by preliminary comparison of fingerprints, more work need to be done for the activity confirmation.

The third stage was the system biology methods, including metabolomics, genomics and proteomics to clarify their therapeutic basis and mechanism of action more clearly [12]. Usually the system biology methods indicate the grade discrimination based on whole metabolites, primary and secondary metabolites, low and high molecular compounds, and volatile and nonvolatile compounds contained in the herbal formula. However, these methods are also proved to be timeconsuming, arduous, and difficult for clarifying the synergistic action of CHM and only show the metabolomics aspects of those herbs, but not link with their efficacy. The main problems to control the curative effects and systematic effects of the real activity of CHMs still exist. The lack of correlation between chemical compositions and clinical efficacy is the key issue to be solved.

\section{Traditional CHM Based New Drug Development Process}

Nowadays approximately $80 \%$ of antimicrobial, cardiovascular, immunosuppressive, and anticancer drugs are originated from herbal medicine [13]. And, actually, about half of the pharmaceuticals are derived from compounds firstly identified from herbs as active ingredients [14]. Among herbal medicine, CHM are mainly made of formula from a combination of Chinese herbs which contains hundreds of active ingredients. These ingredients will be potentially useful for the development of therapeutic agents. The traditional CHM based new drug development process usually includes six steps as listed in Figure 1.

\section{Efficacy-driven Quality Control Platform Framework}

Our research team has been studied a Chinese herbal proprietary medicine (MaZiRenWan, MZRW) for ten years [15]. Although MZRW has been widely used in clinical practice and was proved to be effective in our previous studies, the total active components of MZRW are still unknown. On the other hand, the present quality control studies of MZRW are still based on simply evaluating the content of several main compounds of MZRW, while these main compounds may have no activity. Therefore, more work need to be done to explore the active compounds of MZRW, and a systematic efficacy-driven quality control platform of MZRW need to be established based on the content of real active components.

Based on our previous work, we will establish a systematic quality control platform for Chinese herbal formula from raw material to final

${ }^{*}$ Corresponding author: Zhao-xiang Bian, School of Chinese Medicine, Hong Kong Baptist University, 3/F, Jockey Club School of Chinese Medicine Building, 7 Baptist University Road, Kowloon Tong, Hong Kong SAR, E-mail: bzxiang@hkbu.edu.hk

Received March 01, 2014; Accepted March 24, 2014; Published March 27, 2014

Citation: Zhong LLD, Hu DD, Xu HX, Han QB, Bian ZX (2014) Efficacy-Driven Quality Control Platform for Chinese Herbal Medicine. Pharm Anal Acta 5: 291. doi: 10.4172/2153-2435.1000291

Copyright: (c) 2014 Zhong LLD, et al. This is an open-access article distributed under the terms of the Creative Commons Attribution License, which permits unrestricted use, distribution, and reproduction in any medium, provided the original author and source are credited. 


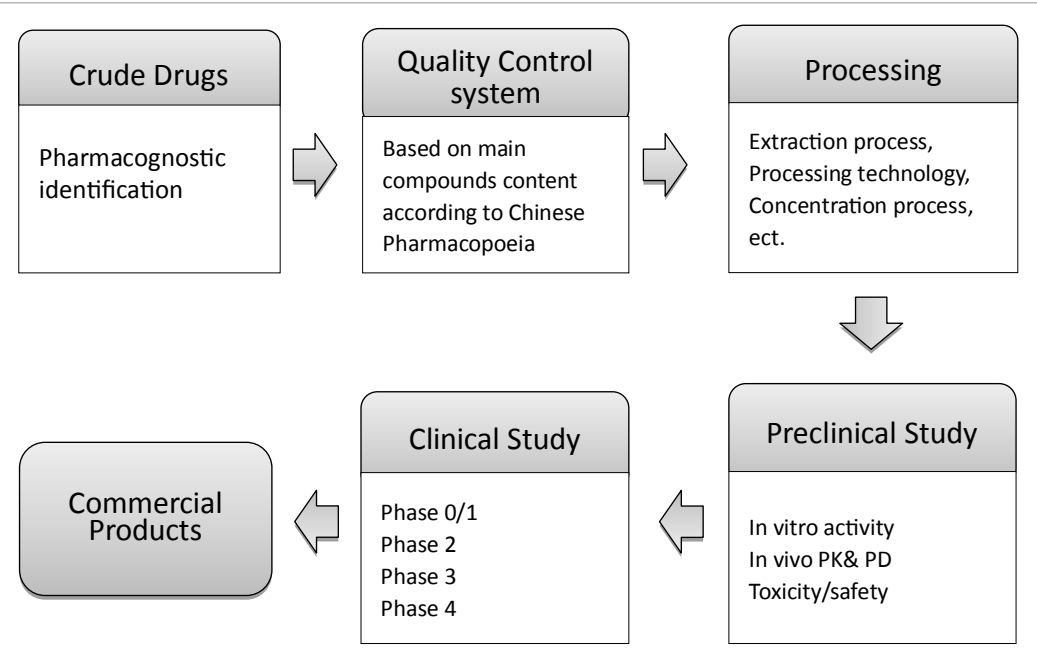

Figure 1: The traditional CHM based new drug development process.

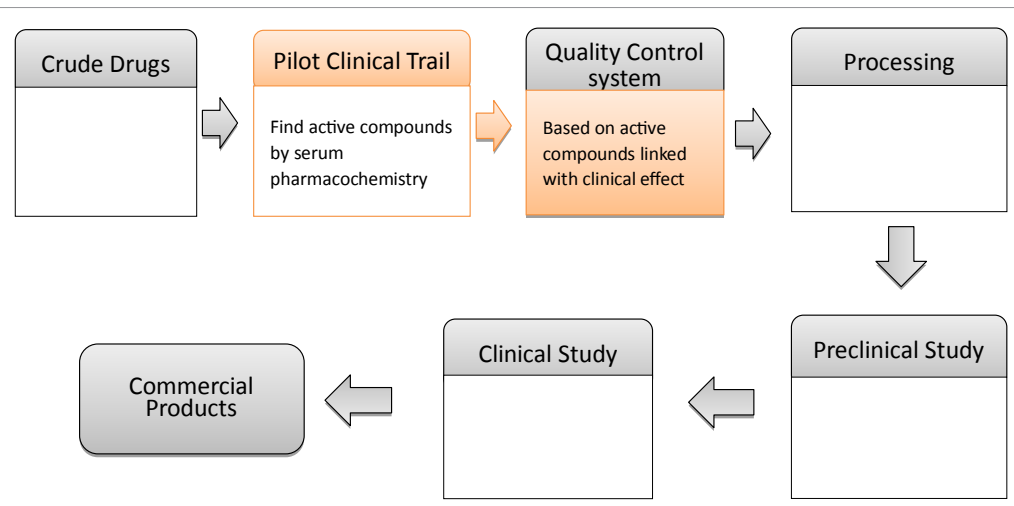

Figure 2: Crude drugs-pilot clinical trial-quality control system based on clinicaltrial-processingpreclinical study-clinical study-commercialproducts.

product based on bio-activity assessments through clinical sample evaluation and pharmacological confirmation, thus to provide a revolutionary approach consolidated reference for the quality control of Chinese herbal formula. We suggest two key elements of this framework: (i) CHM based new drug discovery should include a pilot clinical study to confirm its efficacy and safety and identify the compounds absorbed into blood and metabolic profiling of CHM in human subjects; (ii) The quality control platform should be combined with the data from the pilot clinical study which the targeted components should be connected with the efficacy. With the integration of the new model, the proposed process will include seven steps (Figure 2).

The new framework of the efficacy-driven quality control platform will mainly include the phases as follows: (i) to identify the compounds absorbed into blood and metabolic profiling of CHM in human subjects through a pilot clinical study; (ii) to compare the effects of compounds between absorbed or not absorbed into blood in animal samples; (iii) to determine the pharmacological effects of compounds identified from human samples; (iv) to set up a quality control system based on the compounds which links with pharmacological effects; (v) to compare clinical efficacy in human samples of the new quality control system with conventional quality control method.

Ultimately, our key recommendations are as follows: firstly, the quality control of CHM must be connected with clinical efficacy; secondly, a pilot clinical trial to evaluate the isolated components should be provided; finally, the identified components should be determined through pharmacological experiments.

\section{References}

1. Pan SY, Zhou SF, Gao SH, Yu ZL, Zhang SF, et al. (2013) New Perspectives on How to Discover Drugs from Herbal Medicines: CAM's Outstanding Contribution to Modern Therapeutics. Evid Based Complement Alternat Med 2013: 627375 .

2. Fu DL, Lu L, Zhu W, Li JH, Li HQ, et al. (2013) Xiaoxuming decoction for acute ischemic stroke: a systematic review and meta-analysis. J Ethnopharmacol 148: 1-13.

3. Jiang M, Yang J, Zhang C, Liu B, Chan K, et al. (2010) Clinical studies with traditional Chinese medicine in the past decade and future research and development. Planta Med 76: 2048-2064.

4. Xue CC, Zhang AL, Greenwood KM, Lin V, Story DF (2010) Traditional chinese medicine: an update on clinical evidence. J Altern Complement Med 16: $301-$ 312.

5. Li SP, Zhao J, Yang B (2011) Strategies for quality control of Chinese medicines. J Pharm Biomed Anal 55: 802-809.

6. Liang YZ, Xie PS, Chan K (2010) Chromatographic fingerprinting and metabolomics for quality control of TCM. Comb Chem High Throughput Screen 13: $943-953$ 
Citation: Zhong LLD, Hu DD, Xu HX, Han QB, Bian ZX (2014) Efficacy-Driven Quality Control Platform for Chinese Herbal Medicine. Pharm Anal Acta 5: 291. doi: 10.4172/2153-2435.1000291

Page 3 of 3

7. Qu JB, Lou HX, Fan PHF (2005) Application of TLC-bioautographyto drug screening. Chin Tradit Herbal Drugs 36:132-137.

8. Gao H, Wang Z, Li Y, Qian Z (2011) Overview of the quality standard research of traditional Chinese medicine. Front Med 5: 195-202.

9. Li SP, Zhao J, Qian ZM, Li J (2010) Advanced development of chromatography in screening and identification of effective compounds in Chinese material medica. Sci Sin Chim 40:651-667.

10. Shi SY, Zhang YP, Jiang XY, Chen XQ, Huang KL, et al. (2009) Coupling HPLC to on-line, post-column (bio)chemical assays for high-resolution screening of bioactive compounds from complex mixtures. Trends Anal. Chem 28:865-877.

11. Jiang $Y$, David $B$, Tu $P$, Barbin $Y$ (2010) Recent analytical approaches in quality control of traditional Chinese medicines--a review. Anal Chim Acta 657: 9-18.
12. Katoh A, Fukuda S, Fukusaki E, Hashimoto T, Hayasaki T, et al. (2011) Systems biology in a commercial quality study of the Japanese Angelica radix: toward an understanding of traditional medicinal plants. Am J Chin Med 39: 757-777.

13. Gordaliza M (2009) Terpenyl-purines from the sea. Mar Drugs 7: 833-849.

14. Krief S, Martin MT, Grellier P, Kasenene J, Sévenet T (2004) Novel antimalarial compounds isolated in a survey of self-medicative behavior of wild chimpanzees in Uganda. Antimicrob Agents Chemother 48: 3196-3199.

15. Cheng CW, Bian ZX, Zhu LX, Wu JC, Sung JJ (2011) Efficacy of a Chinese herbal proprietary medicine (Hemp Seed Pill) for functional constipation. Am J Gastroenterol 106: 120-129. 